\title{
The Different Dose-Volume Effects of Normal Tissue Complication Probability Using LASSO for Acute Small-Bowel Toxicity during Radiotherapy in Gynecological Patients with or without Prior Abdominal Surgery
}

\author{
Tsair-Fwu Lee ${ }^{1}$ and Eng-Yen Huang ${ }^{2,3}$ \\ ${ }^{1}$ Medical Physics and Informatics Laboratory of Electronics Engineering, National Kaohsiung University of Applied Sciences, \\ Kaohsiung 807, Taiwan \\ ${ }^{2}$ Department of Radiation Oncology, Kaohsiung Chang Gung Memorial Hospital and Chang Gung University College of Medicine, \\ 123 Ta-Pei Road, Niao-Sung District, Kaohsiung 83305, Taiwan \\ ${ }^{3}$ School of Traditional Chinese Medicine, Chang Gung University College of Medicine, Taoyuan 333, Taiwan
}

Correspondence should be addressed to Eng-Yen Huang; huangengyen@gmail.com

Received 10 May 2014; Accepted 30 June 2014; Published 16 July 2014

Academic Editor: Cheng-Shie Wuu

Copyright (C) 2014 T.-F. Lee and E.-Y. Huang. This is an open access article distributed under the Creative Commons Attribution License, which permits unrestricted use, distribution, and reproduction in any medium, provided the original work is properly cited.

Purpose. To develop normal tissue complication probability (NTCP) model with least absolute shrinkage and selection operator (LASSO) to analyze dose-volume effects that influence the incidence of acute diarrhea among gynecological patients with/without prior abdominal surgery. Methods and Materials. Ninety-five patients receiving gynecologic radiotherapy (RT) were enrolled. The endpoint was defined as the grade $2^{+}$acute diarrhea toxicity during treatment. We obtained the range of small-bowel volume in V4 Gy to V40 Gy of dose. Results. The number of patients experiencing grade $2^{+}$acute diarrhea toxicity was $23 / 61$ (38\%) in the group without abdominal surgery (group 0) and 17/34 (50\%) patients with abdominal surgery (group 1). The most significant predictor was found for the logistic NTCP model with V16 Gy as the cutoff dose for group 0 and V40 Gy for group 1. Logistic regression NTCP model parameters were $\mathrm{TV}_{10} \approx 290 \mathrm{cc}$ for V16 Gy and $\mathrm{TV}_{10} \approx 75 \mathrm{cc}$ for V40 Gy, respectively. Conclusion. To keep the incidence of grade $2^{+}$acute small-bowel toxicity below $10 \%$, we suggest that small-bowel volume above the prescription dose (V16 Gy) should be held to $<290 \mathrm{cc}$ for patients without abdominal surgery, and the prescription dose (V40 Gy) should be maintained $<75 \mathrm{cc}$ for patients with abdominal surgery.

\section{Introduction}

Radiation therapy (RT) plays an important role in the treatment of abdominal and pelvic disease. However, radiationinduced acute diarrhea is common side effect which correlates with dose-volume effect of small bowel [1-3]. The acute diarrhea induced by radiotherapy includes increased stool frequency, increased degrees of hardness and volume of stool, severely watery stools, or a mixture of blood, serum, mucus, and other items. Acute diarrhea not only affects the patient's quality of life, but is also serious and requires hospitalization due to dehydration. Severe acute diarrhea has led to the failure of complete therapy or reduction in the total dose, all of which may diminish the effectiveness of therapy [4]. The relationship of the small-bowel dose and irradiative volume with acute diarrhea would make progress in RT strategies and help to direct further efforts to reduce the incidence of this intractable side effect [5].

Prior abdominal surgery may influence the incidence of acute diarrhea, as has been reported in previous studies [5-7]. The relationship between the volume of small bowel irradiated and the degree of acute small-bowel toxicity experienced has been well recognized but poorly quantified. In this work, we introduce the normal tissue complication probability (NTCP) model to quantify the relationship between the incidence of acute diarrhea and dose-volume effects of 
small bowel, which potentially identify a more specific dosevolume relationship.

NTCP modelling in radiation therapy aims to describe the correlation between dosimetric parameters and the probability of side effects [8-10]. We can assign the great information about inhomogeneous dose distributions and corresponding outcome data in large patient populations into few-parametric models [11]. This single probability value is more clearly able to reveal the relationship between dose volume and acute diarrhea in an individual treatment plan.

The least absolute shrinkage and selection operator (LASSO) is based on shrinkage estimated and has been widely used in the statistics field. Xu et al. [12,13] introduced LASSO to build NTCP models of xerostomia after three-dimensional conformal radiation therapy (3D-CRT) for head and neck cancer. The advantages of LASSO include (1) a smaller mean squared error (MSE) than conventional methods; (2) handling the multicollinearity problem; (3) overall variable selection; and (4) coefficients shrink [12, 13]. In addition, being easy to implement is one of the merits that attract users. $\mathrm{Xu}$ et al. recommended the LASSO method for the NTCP predictive factor selection $[12,14]$.

Validated NTCP models of small-bowel toxicity are needed to provide evidence for dosimetric guidelines in treatment planning and protocols [15]. Previous studies have been reported about dose-volume parameters and acute gastrointestinal toxicity in various pelvic malignancies. However, NTCP models describing RT-induced acute diarrhea in gynecologic cancer, particularly taking into account patients with/without prior abdominal surgery, are lacking. Therefore, the purpose of this study was to develop a logistic regression NTCP model with LASSO to make valid predictions about the incidence of acute diarrhea among gynecological patients.

\section{Materials and Methods}

2.1. Patient Characteristics. The study population was composed of 95 patients receiving gynecologic radiotherapy. Patients were grouped into without (group 0) or with (group 1) prior abdominal surgery. Those patients were initially evaluated with pathologic assessment, physical examination, routine laboratory work, abdominal-pelvic CT scan, and chest X-ray. All patients were treated by a single radiation oncologist (E.Y.H.). Patients with a history of intestinal diseases such as inflammatory bowel disease, diverticulitis, and autoimmune diseases were excluded. The study was under the proof of the institutional review board of the hospital (97-1370B). Patient characteristics are summarized in Table 1.

2.2. Bowel Delineation. To accurately calculate irradiated small-bowel volume, patients received computed tomography simulation in the supine position, with a thermoplastic body cast for immobilization, an emptied bladder and bowel, and with small-bowel contrast (4\% Gastrografin). The patients were scanned from the mid-abdomen to $10 \mathrm{~cm}$ below the ischial tuberosities in $0.5 \mathrm{~cm}$ increment slices. Therefore,
TABLE 1: Characteristics of patients.

\begin{tabular}{|c|c|c|c|}
\hline & $\begin{array}{c}\text { Group 0 } \\
(n=61) \\
\text { Number }(\%)\end{array}$ & $\begin{array}{c}\text { Group 1 } \\
(n=34) \\
\text { Number }(\%)\end{array}$ & $P$ value \\
\hline Age (y) & & & 0.031 \\
\hline Mean & 60.1 & 54.9 & \\
\hline Range & $29-90$ & $32-75$ & \\
\hline$<50$ & $16(26)$ & $9(26)$ & \\
\hline $51-60$ & $18(30)$ & $15(45)$ & \\
\hline $61-70$ & $11(18)$ & $9(26)$ & \\
\hline$>70$ & $16(26)$ & $1(3)$ & \\
\hline BMI & & & 0.360 \\
\hline Mean & 24.9 & 25.7 & \\
\hline Range & $16.6-35.1$ & $20.1-34.5$ & \\
\hline$<20$ & $8(13)$ & $0(0)$ & \\
\hline $20-26$ & $27(44)$ & $20(59)$ & \\
\hline$>26$ & $26(43)$ & $14(41)$ & \\
\hline Stage & & & 0.007 \\
\hline I-II & $52(85)$ & $19(56)$ & \\
\hline III-IV/recurrent & $9(15)$ & $15(44)$ & \\
\hline Diabetes & & & 0.763 \\
\hline No & $52(85)$ & $30(88)$ & \\
\hline Yes & $9(15)$ & $4(12)$ & \\
\hline Hypertension & & & 0.779 \\
\hline No & $50(82)$ & $29(85)$ & \\
\hline Yes & $11(18)$ & $5(15)$ & \\
\hline Chemoradiotherapy & & & 0.013 \\
\hline No & $15(25)$ & $17(50)$ & \\
\hline Yes & $46(75)$ & $17(50)$ & \\
\hline External beams doses (Gy) & & & 0.469 \\
\hline WP 19.8, PM/LP 32.4 & $0(0)$ & $1(2.9)$ & \\
\hline WP 39.6 & $20(33)$ & $5(14.7)$ & \\
\hline WP 39.6, PM/LP 45 & $27(44)$ & $23(67.6)$ & \\
\hline WP 39.6, LP 50.4 & $0(0)$ & $2(5.9)$ & \\
\hline WP 45 & $4(7)$ & $1(2.9)$ & \\
\hline WP 45, PM/LP 504 & $10(16)$ & $2(5.9)$ & \\
\hline
\end{tabular}

Group 0: patients without prior abdominal surgery; group 1: patients with prior abdominal surgery; BMI: body mass index; WP: whole pelvis; LP: low pelvis; PM: parametrium.

Differences between the group 0 and group 1 cohort were described with an independent sample $t$-test for continuous variables and chi-square test for dichotomous variables.

the volumetric data were transferred to the Pinnacle treatment planning system (Philips, Fitchburg, WI).

The small bowel was contoured from the L4-5 interspace to its lowest extent in the pelvis. The outermost extent of the contrast-enhanced small-bowel loops was outlined on each axial CT slice. The small bowel in the upper abdomen was not included. A sample to illustrate the delineation of the bowels is shown in Figure 1. The dose-volume relationship was calculated in the planning system. The dose was prescribed to the isodose curve (95\% to $100 \%$ ) that surrounded the treatment 


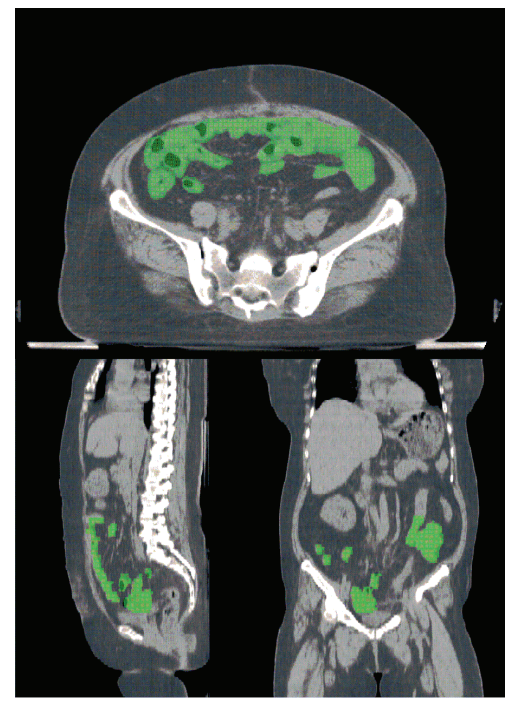

Figure 1: The delineation of the bowels.

volume at risk. The onset and grade of diarrhea during wholepelvic irradiation were recorded as small-bowel toxicity up to $39.6 \mathrm{~Gy}$ in 22 fractions.

2.3. Treatment Technique. The RT treatment technique typically used opposed posterior to anterior and opposed lateral field arrangement for the whole pelvis [6] to fulfil the homogeneity requirement. External whole-pelvic irradiation was initially administered with photons from a 10 or $15 \mathrm{MV}$ linear accelerator (Varian Medical Systems, Palo Alto, CA) with a four-field technique. The dose per fraction was $1.8 \mathrm{~Gy}$ in 5 fractions weekly. The planning dose was 39.6-45 Gy in $22-25$ fractions. An additional boost (5.4-9 Gy in 3-5 fractions) was given to the bilateral parametrial and pelvic wall through anteroposterior/posteroanterior ports with a $4 \mathrm{~cm}$ central shielding in patients with greater than Stage IIA cervical cancer without hysterectomy. In patients with hysterectomy, the boost doses (5.4-9 Gy in 3-5 fractions) to low pelvis were delivered. The fields and doses beyond 39.6 Gy are shown in Table 1.

2.4. Brachytherapy. High-dose-rate intracavitary brachytherapy through a remote after-loading system (microSelectron; Nucletron, Veenendaal, The Netherlands) using 192Ir sources was given. The prescribed dose of brachytherapy was either 4.5 or $6 \mathrm{~Gy}$ at Point A and $27 \mathrm{~Gy}$ in 6 fractions or $24 \mathrm{~Gy}$ in 4 fractions. In vaginal intracavitary brachytherapy, we delivered 8-15 Gy in 2-5 fractions.

2.5. Gastrointestinal Toxicity. The severity of acute diarrhea during treatment was graded using the common toxicity criteria (CTC) up to $39.6 \mathrm{~Gy}$ in 22 fractions [16]: grade 0 (none), grade 1 (an increase from 2 to 3 stools per day over pretreatment), grade 2 (an increase of 4-6 stools per day or nocturnal stools), grade 3 (increase of $>7$ stools per day or incontinence or need for parenteral support for dehydration), and grade 4 (physiologic consequences requiring intensive care or hemodynamic collapse).

According to previous studies, which suggested that gastrointestinal drugs may influence bowel symptoms, we managed patients with gastrointestinal symptoms by using a uniform protocol, patients with CTC: grade 1 diarrhea (mebeverine was prescribed), grade 2 diarrhea (loperamide was added), grade 3 diarrhea (loperamide was prescribed three times a day), and grade 4 diarrhea (hospitalization and radiotherapy were interrupted until diarrhea downgraded to grade 2 diarrhea or less).

In this study, we investigated the NTCP predictive models for the incidence of acute diarrhea. The severity of diarrhea was graded into four levels. In our data, there were no patients suffering with grade 4 diarrhea, and we classified grade 2 and greater diarrhea (grade $2^{+}$) as clinically significant.

2.6. Dose-Volume Response Modeling. The cutting endpoints (grade $2^{+}$acute diarrhea toxicities) were analyzed using logistic regression NTCP analysis with an extended bootstrapping technique, as described by El Naqa et al. [17] and Beetz et al. $[8,10]$. We obtained the range of small-bowel volume in doses from V4 Gy to V40 Gy, at $10 \%$ intervals with the checkpoint dose $39.6 \mathrm{~Gy}$ ( $\approx 40 \mathrm{~Gy}$ ). The most significant dosevolume predictive factor for the logistic regression model was determined by using the LASSO with bootstrapping technique $[8,10]$. The LASSO was first proposed by Tibshirani in 1996; details can be found in $[12,18-20]$. It uses the following equation to shrink the coefficients and select the predictive factors:

$$
\underset{\beta}{\operatorname{argmin}}\|Y-X \beta\|^{2} \text { subject to }\|\beta\|=\sum_{j=0}^{d}\left|\beta_{j}\right| \leq t,
$$

where $d$ is the number of variables selected and $t$ is tuning parameter that controls the degree of penalty $[12,21]$. To account for the overfitting problem, two datasets were used, 


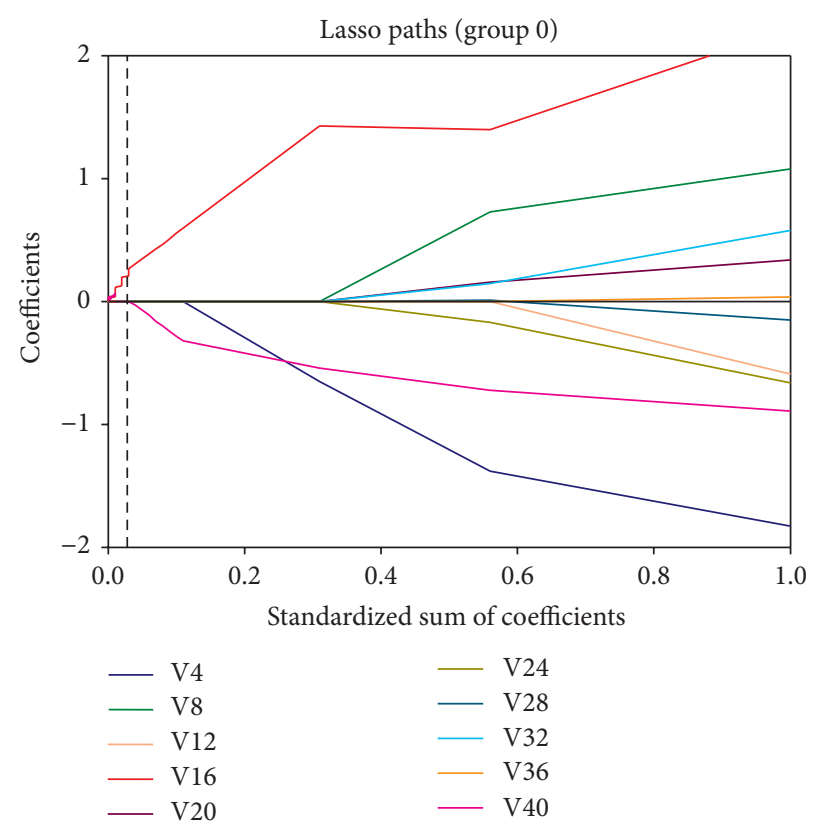

(a)

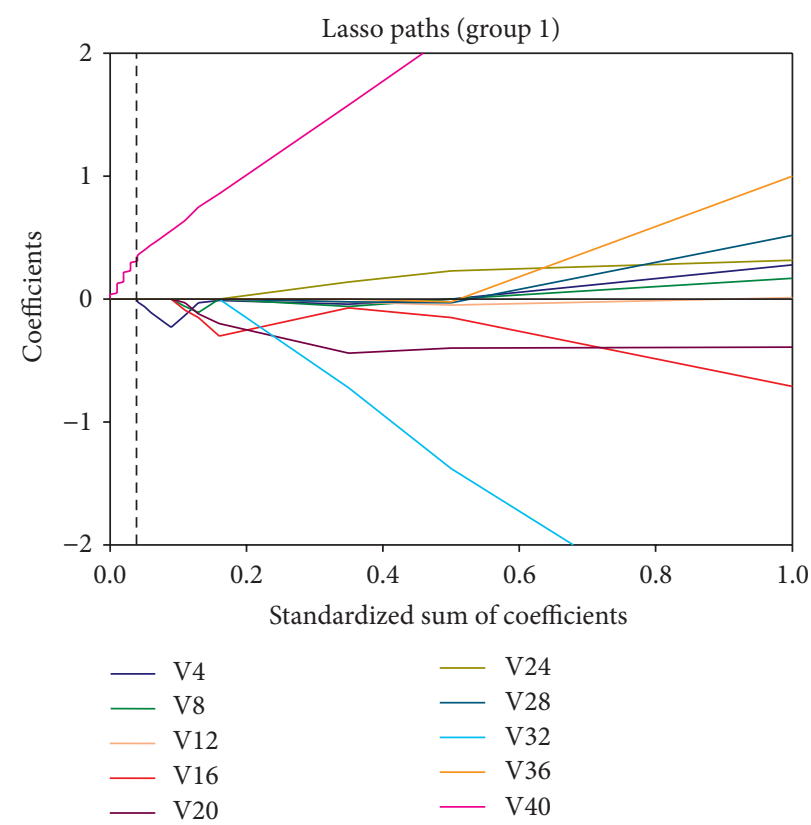

(b)

FIGURE 2: The LASSO shrinking path diagrams for the dosimetric candidate predictive factors in group 0 (a) and group 1 (b), respectively. LASSO: least absolute shrinkage and selection operator; group 0: patients without prior abdominal surgery; group 1: patients with prior abdominal surgery; V4 V40: the range of small-bowel volume in $x$ Gy of dose.

that is, a training set and a test set; a model was built based on a training set and fitted to the training set itself and also tested with a test set. We used nested 10-fold cross validation to obtain the optimum predictive factors $[12,14$, 19]. The model with medium AUC performance was selected as the optimum model with the most significant dose-volume predictive factor.

Toxicity and dose-volume predictive data were then fit to a logistic NTCP function:

$$
\mathrm{NTCP}=\left[1+\left(\frac{\mathrm{TV}_{50}}{V}\right)^{4 \gamma}\right]^{-1},
$$

where $V$ is the volume of small bowel receiving a given dose level, $\mathrm{TV}_{50}$ is the tolerance volume corresponding to $50 \%$ incidence of complications, and $\gamma$ is the normalized slope of the volume response curve. The best-fitting values of model parameters were determined using maximum likelihood analysis and the 95\% confidence intervals were found using the profile likelihood method.

The system performance and calibration were evaluated by the AUC, Brier score, $R^{2}$, Omnibus test, and HosmerLemeshow test $[8,9]$. Statistical analyses were performed using SPSS 19.0 (SPSS, Chicago, IL).

\section{Results}

Ninety-five patients were included in the analysis. We classified patients into two groups. Patients without prior abdominal surgery were named group $0(n=61)$. They all had cervical cancer. Those who had undergone prior abdominal surgery were named group $1(n=34)$. Their diseases included cervical cancer, endometrial cancer, and uterine/ adnexa sarcoma. During radiotherapy, seven patients (11\%), thirty-one (51\%), seventeen (28\%), and six (10\%) patients in group 0 had grades $0,1,2$, and 3 diarrhea, respectively. Eight patients (23.5\%), nine (26.5\%), nine (26.5\%), and eight $(23.5 \%)$ patients in group 1 had grades $0,1,2$, and 3 diarrhea, respectively. No patients suffered from grade 4 diarrhea. The number of patients experiencing grade $2^{+}$ acute diarrhea toxicity was $23 / 61(38 \%)$ in the group without abdominal surgery and $17 / 34$ (50\%) in those with abdominal surgery.

The most significant dose-volume predictive factor for the logistic regression NTCP model was determined by using the LASSO with bootstrapping technique (the LASSO shrinking path diagrams are shown in Figure 2). We used 300 bootstraps for each analysis. The initial dosimetric candidate predictive factors were shown in Table 2 . The most significant predictor was found for the logistic regression NTCP model with V16 Gy as the cutoff dose for group 0 and V40 Gy for group 1, respectively.

The fitted dose-response curves (logistic NTCP model) for the incidence of grade $2^{+}$acute diarrhea toxicity for the gynecological patients with/without prior abdominal surgery are shown in Figure 3. NTCP fitted parameters were $\mathrm{TV}_{50}=$ $409.4 \mathrm{cc}$ (CI: 391.1-427.7 cc), $\gamma=1.92$ (CI: 1.36-2.62) and $\mathrm{TV}_{50}=99.0 \mathrm{cc}(\mathrm{CI}: 96.6-101.5 \mathrm{cc}), \gamma=2.34$ (CI: 1.85-2.83) for the patients in groups 0 and 1 , respectively. And $\mathrm{TV}_{10} \approx 290 \mathrm{cc}$ for $\mathrm{V} 16 \mathrm{~Gy} ; \mathrm{TV}_{10} \approx 75 \mathrm{cc}$ for $\mathrm{V} 40 \mathrm{~Gy}$, respectively $\left(\mathrm{TV}_{10}\right.$ is the tolerance volume corresponding to $10 \%$ incidence of complications). 
TABLE 2: Dosimetric candidate predictive factors initially.

\begin{tabular}{|c|c|c|c|c|c|c|}
\hline \multirow{2}{*}{ Volumes } & \multicolumn{3}{|c|}{ Group 0} & \multicolumn{3}{|c|}{ Group 1} \\
\hline & Range & Median & Logistic correlation & Range & Median & Logistic correlation \\
\hline V4 Gy & $76-990$ & 460 & -0.009 & $65-772$ & 329 & -0.039 \\
\hline V8 Gy & $65-889$ & 422 & 0.016 & $39-700$ & 297 & 0.043 \\
\hline V12 Gy & $57-824$ & 385 & -0.094 & $27-665$ & 276 & -0.042 \\
\hline V16 Gy & $51-772$ & 348 & 0.166 & $20-634$ & 255 & 0.050 \\
\hline V20 Gy & $15-721$ & 230 & -0.003 & $13-603$ & 175 & -0.021 \\
\hline V24 Gy & $6-678$ & 177 & -0.001 & $12-578$ & 135 & 0.026 \\
\hline V28 Gy & $4-651$ & 138 & -0.033 & $10-324$ & 111 & -0.023 \\
\hline V32 Gy & $3-611$ & 115 & 0.055 & $8-296$ & 102 & 0.069 \\
\hline V36 Gy & $1-584$ & 104 & 0.024 & $4-274$ & 96 & -0.407 \\
\hline V40 Gy & $0-545$ & 89 & -0.063 & $1-249$ & 77 & 0.740 \\
\hline
\end{tabular}

V4 V40 Gy: the range of small-bowel volume in $x$ Gy of dose; group 0: patients without prior abdominal surgery; group 1: patients with prior abdominal surgery.

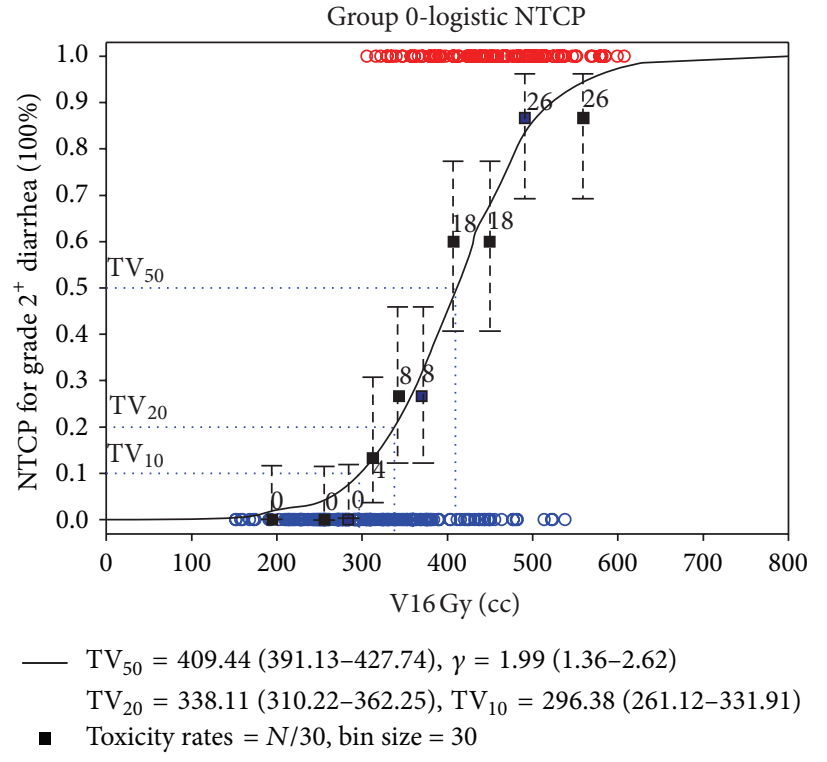

(a)

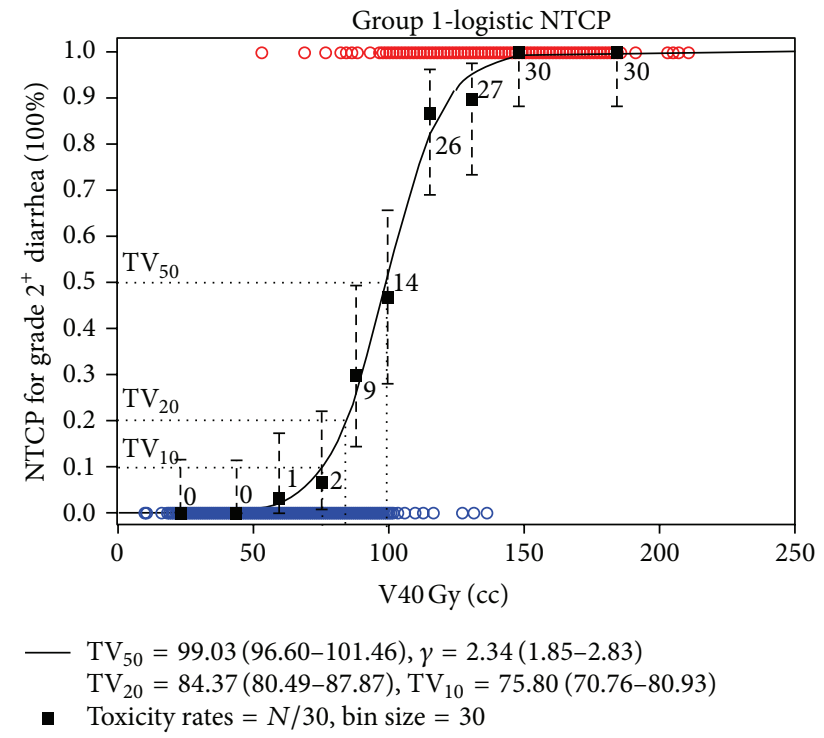

(b)

FIGURE 3: The logistic normal tissue complication probability model with V16 Gy as the cutoff dose for group 0 (a) and V40 Gy for group 1 (b). $\mathrm{TV}_{50}$ is the tolerance volume corresponding to $50 \%$ incidence of complications, and $\gamma$ is the normalized slope of the volume response curve.

The overall performance and calibration for the NTCP models were satisfactory and corresponded well with the expected values (Table 3). The AUC for the optimal model was 0.90 (range 0.79-0.95) and 0.96 (range 0.91-0.99) in patients without and with previous abdominal surgery, respectively. Finally, the calibration slope of $\geq 0.99$ for both models showed a significant agreement between predicted risk and observed outcome for both LASSO NTCP models (Figure 4).

\section{Discussions}

Many researches have discussed the different dose population of gastrointestinal tract between preoperative and postoperative radiotherapy. Shadad et al. thought that previous abdominal surgery increases the risk of radiation toxicity because anatomical changes that increase intestinal exposure to radiation such as postoperative small intestine prolapse into the pelvic cavity or surgical adhesions that fix intestinal segments within the radiation field can all predispose part of the intestine to receive higher doses of radiation [22]. Robertson et al. [5] noted that the incidence of grade 3 diarrhea is higher in rectal cancer patients with abdominal surgery than in those without abdominal surgery, with rates of $28 \%$ and $18 \%$, respectively. Recently, they reported a similar result; this group found that the incidences of diarrhea in patients with and without prior abdominal surgery were $29 \%$ and $14 \%$, respectively [23]. The corresponding rates were $23 \%$ and $10 \%$ in the present study, showing a lower incidence of grade 3 diarrhea. The reason for this may be 
TABLE 3: System performance evaluation.

\begin{tabular}{lcccccr}
\hline Group & Cutoff dose & AUC (range) & Accuracy (range) & $R^{2}$ & Brier & $\begin{array}{c}\text { Slope of calibration } \\
\text { curve }\end{array}$ \\
\hline 0 & V16 Gy & $0.90(0.79-0.95)$ & $0.81(0.70-0.87)$ & 0.98 & 0.10 & 0.99 \\
1 & V40 Gy & $0.96(0.91-0.99)$ & $0.90(0.83-0.93)$ & 0.99 & 0.07 & 0.14 \\
\hline
\end{tabular}

AUC: area under the receiver operating characteristic curve; HL: Hosmer-Lemeshow test.

Group 0: patients without prior abdominal surgery; group 1: patients with prior abdominal surgery.

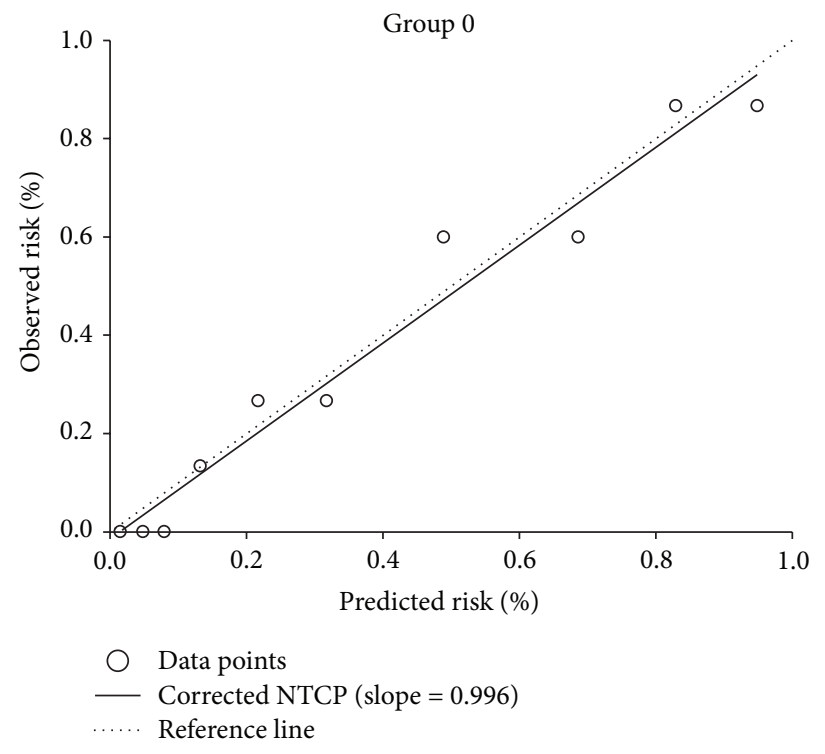

(a)

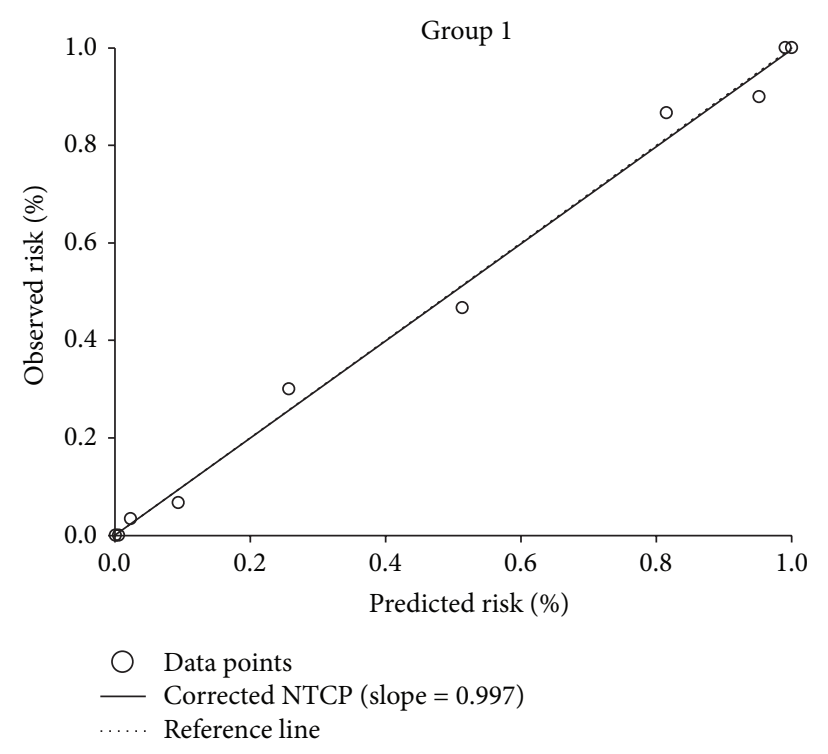

(b)

FIGURE 4: Calibration of a predictive model for patients in group 0 (a) and for patients in group 1 (b), respectively.

related to different cancers. No rectal disease was noted in our patients. However, rectal function impairments were noted in patients with rectal mass (preoperative) or rectal resection (postoperative) [23]. Therefore, using CTC grading, patients with rectal cancer may have more severe diarrhea than our gynecological patients. Furthermore, we noted no larger V4V40 Gy in group 1 than group 0 patients (Table 2). However, more patients developed grade 2-3 diarrhea. Therefore, we confirmed the importance of abdominal surgery on acute diarrhea.

Although patients in group 0 had larger small-bowel volumes irradiated than patients in group 1 . (Table 2), the diarrhea rate was higher in group 1 patients. This points out the importance of abdominal surgery on dosimetry and toxicity of the small bowel.

Group 0 patients had an older age than those in group 1 (Table 1). Based on our prior study [24], small-bowel volumes are larger than in elder patients. We do not know the cause of this phenomenon. In patients with an intact uterus, atrophic uterine may mimic hysterectomy and also increases the volume of small bowel irradiated. Therefore, the old age effect may explain why group 0 patients had larger smallbowel volume than group 1 patients. Because elder patients account for the majority of patients undergoing definitive radiotherapy, their small-bowel volume may enlarge and dose constraints (V40 Gy $<75 \mathrm{cc}$ or V16 Gy $<290 \mathrm{cc}$ ) are very important for using IMRT.

The most significant dose-volume predictive factor for the logistic regression NTCP model can be determined by using statistical analysis. Many researchers used the log likelihood (LL), average likelihood, stepwise selection, Bayesian information criterion (BIC), and Akaike information criterion (AIC) to deal with this topic [8]. Xu et al. [12] showed that their NTCP models for xerostomia developed with LASSO have considerably better prediction performance than the commonly used stepwise selection method. Early NTCP models, like the LKB [25] and the univariate logistic regression model [26, 27], are based on information derived from dose-volume histograms generated from dose distributions in the target volumes and the surrounding organs that are at risk. If the models developed using V4-V40 Gy individually did not take into account abdominal surgery; the AUC values for the model were below 0.65 . We considered the importance of abdominal surgery on acute diarrhea and quantified the relationship between the incidence of acute diarrhea and dose-volume effects of the small bowel. The most significant dose-volume predictive factor for the logistic regression NTCP model was determined by the LASSO with 
V16 Gy as the cutoff dose for group 0 and V40 Gy for group 1, respectively. The system performance AUC values improved from 0.65 to 0.90 for group 0 and from 0.58 to 0.96 for group 1 , respectively. We suggest that this is useful for clinical practice in consideration of time and cost efficiency; overall variable selection and coefficients shrink were performed in one step while handling the multicollinearity problem $[12,19,28]$.

Many studies have discussed the dose-volume effects. Baglan et al. reported on 40 patients with rectal cancer treated with the four-field technique. Eight patients (20\%) did not receive abdominal surgery before radiotherapy. The small-bowel volume receiving at least $15 \mathrm{~Gy}$ (V15 Gy) was the best predictor [4]. Tho et al. noted that small-bowel volume correlated strongly with severity of diarrhea at $15 \mathrm{~Gy}$ (V15 Gy) $<100 \mathrm{cc}$ in 41 patients undergoing preoperative chemoradiotherapy for rectal cancer [7]. Several other studies have reported dose-volume relationships for the bowel in patients treated for rectal cancer with four-field techniques $[1,5]$. They all found a high correlation of $15 \mathrm{~Gy}$ (V15 Gy) as a predictor of small-bowel toxicity. The quantitative analysis of normal tissue effects in the clinic (QUANTEC) review summarizes the available $3 \mathrm{D}$-CRT data to update and refine the normal tissue dose/volume tolerance guidelines for small bowel which is "the absolute volume of small bowel receiving $\geq 15 \mathrm{~Gy}$ should be held to $<120 \mathrm{cc}$ when possible to minimize severe acute toxicity $<10 \%$, if delineating the contours of bowel loops themselves" $[29,30]$. In this study, we found that to keep the incidence of grade $2^{+}$acute small-bowel toxicity below $10 \%$, the small-bowel volume above the prescription dose V16 Gy should be maintained at $<290 \mathrm{cc}$ for patients without prior abdominal surgery; and the prescription dose V40 Gy should be held at $<75 \mathrm{cc}$ for patients with prior abdominal surgery. The difference in volume constraints $(<120 \mathrm{cc}$ versus $<290 \mathrm{cc}$ ) on 15-16 Gy level may be dependent on grade of diarrhea (grade $3^{+}$versus grade $2^{+}$) and rectal cancer (yes versus no), respectively. In addition to small bowel, the rectum is the other organ at risk for acute diarrhea. In rectal cancer patients, diarrhea is sensitive to small volumes (V15 Gy) of the small bowel because of rectal function impairment.

To our knowledge, recently, there has been no standard dose-volume constraint for the volume or percentage of irradiated small bowel in gynecological patients. Therefore, the aim of the present study was to develop specific NTCP values. Huang et al. [6] showed that gynecologic patients without and with abdominal surgery have different volume effects on small-bowel toxicity during whole-pelvic irradiation. Lowdose volumes can be used as a predictive index of grade $2^{+}$diarrhea in patients without abdominal surgery. The fulldose volume is more important than low-dose volume for grade $2^{+}$diarrhea in patients with abdominal surgery. In this study, we confirmed that and determined the most significant dose-volume predictive factor by using the LASSO with bootstrapping technique. Our finding showed the most significant predictor for the logistic NTCP model with V16 Gy as the cutoff dose for patients without prior abdominal surgery and V40Gy for patients with prior abdominal surgery. Logistic regression NTCP model parameters were found: approximately $\mathrm{TV}_{50}=400 \mathrm{cc}$ for V16 Gy and $\mathrm{TV}_{50}=100 \mathrm{cc}$ for V40 Gy, respectively.
The fact that chemotherapy, a nondosimetric patient factor, may affect the risk of acute diarrhea toxicity is an issue of special concern. In this study, during radiotherapy with concurrent chemotherapy, the number of patients experiencing grade $2^{+}$acute diarrhea toxicity was $18 / 46$ (39\%) in the group without abdominal surgery (group 0) and $11 / 17(65 \%)$ in those with abdominal surgery (group 1). The incidences of toxicity differences between these two cohorts were presented $(P=0.013)$. Chitapanarux et al. found that grade 1-2 acute and late diarrhea were observed in 20 and $40 \%$ caused by chemotherapy, respectively, in their thirty metastatic or recurrent cervical cancer patients [31]. Moore et al. studied patients receiving pelvic RT and showed that seven were treated with weekly cisplatin at doses of 30 and $40 \mathrm{mg} / \mathrm{m}^{2}$, with one dose-limiting toxicity, that is, febrile neutropenia with grade 3 diarrhea [32]. Rose et al. reported that eleven patients received sixty-three doses of the topotecan/cisplatin combination therapy, nine with grade 2 diarrhea toxicity during pelvic radiation therapy in patients with locally advanced cervical cancer [33]. These reports show that the use of chemotherapy was typically correlated with acute diarrhea toxicity risk. In addition, no significant difference for the small-bowel volume irradiated was observed in the patient groups with/without concurrent chemotherapy, $P>0.52$ for group 0 and $P>0.28$ for group 1, respectively. Further studies may be necessary to evaluate what regimen for chemotherapy against acute diarrhea toxicity is better during RT.

There are a number of potential limitations of this study. van der Schaaf et al. [13] reported that approximately 200 patients are needed to obtain a model with high predictive power. In this study, the number of patients assessed for grade $2^{+}$acute diarrhea toxicities was under the recommended 200 patients. Therefore, a larger study sample is required to demonstrate the independent association of these NTCP models with the risk of grade $2^{+}$acute diarrhea toxicity. Moreover, treatment methods may differ among nations and institutions. Differences in radiation modality may create different kinds and different levels of acute diarrhea toxicity. The risk of small-bowel acute diarrhea may be influenced by the techniques used for treatment or factors other than dose, such as the baseline patient risk factors, the coirradiation of other organs, or the fact that bowel motion may be needed for further investigation.

\section{Conclusions}

The LASSO NTCP model can be used to predict the risk of grade $2^{+}$acute diarrhea toxicity. This result illustrates that gynecologic patients with or without prior abdominal surgery have different volume effects on small-bowel toxicity. We suggest a dose-volume constraint for the volume of bowel that can be irradiated.

\section{Conflict of Interests}

The authors declare that there is no conflict of interests regarding the publishing of this paper. 


\section{Acknowledgments}

The authors thank Jing-Chuan Jiang, Nien-Yuan Sung, HsiaoFei Lee, Hui-Min Ting, and Pei-Ju Chao for their technical support. This study was supported financially, in part, by Grants from Chang-Gung Medical Research Project (CMRPG871101), NSC-101-2221-E-151-007-MY3, and NSC-102-2221E-182A-002.

\section{References}

[1] C. Fiorino, R. Valdagni, T. Rancati, and G. Sanguineti, "Dosevolume effects for normal tissues in external radiotherapy: pelvis," Radiotherapy and Oncology, vol. 93, no. 2, pp. 153-167, 2009.

[2] I. Barillot, E. Tavernier, K. Peignaux et al., "Impact of post operative intensity modulated radiotherapy on acute gastrointestinal toxicity for patients with endometrial cancer: results of the phase II RTCMIENDOMETRE French multicentre trial," Radiotherapy and Oncology, vol. 111, no. 1, pp. 138-143, 2014.

[3] D. R. Simpson, W. Y. Song, V. Moiseenko et al., "Normal tissue complication probability analysis of acute gastrointestinal toxicity in cervical cancer patients undergoing intensity modulated radiation therapy and concurrent cisplatin," International Journal of Radiation Oncology, Biology, Physics, vol. 83, no. 1, pp. e81-e86, 2012.

[4] K. L. Baglan, R. C. Frazier, D. Yan, R. R. Huang, A. A. Martinez, and J. M. Robertson, "The dose-volume realationship of acute small bowel toxicity from concurrent 5-FU-based chemotherapy and radiation therapy for rectal cancer," International Journal of Radiation Oncology Biology Physics, vol. 52, no. 1, pp. 176-183, 2002.

[5] J. M. Robertson, D. Lockman, D. Yan, and M. Wallace, "The dose-volume relationship of small bowel irradiation and acute grade 3 diarrhea during chemoradiotherapy for rectal cancer," International Journal of Radiation Oncology Biology Physics, vol. 70, no. 2, pp. 413-418, 2008.

[6] E.-Y. Huang, C.-C. Sung, S.-F. Ko, C.-J. Wang, and K. D. Yang, "The different volume effects of small-bowel toxicity during pelvic irradiation between gynecologic patients with and without abdominal surgery: a prospective study with computed tomography-based dosimetry," International Journal of Radiation Oncology, Biology, Physics, vol. 69, no. 3, pp. 732739, 2007.

[7] L. M. Tho, M. Glegg, J. Paterson et al., "Acute small bowel toxicity and preoperative chemoradiotherapy for rectal cancer: investigating dose-volume relationships and role for inverse planning," International Journal of Radiation Oncology Biology Physics, vol. 66, no. 2, pp. 505-513, 2006.

[8] I. Beetz, C. Schilstra, F. R. Burlage et al., "Development of NTCP models for head and neck cancer patients treated with threedimensional conformal radiotherapy for xerostomia and sticky saliva: the role of dosimetric and clinical factors," Radiotherapy and Oncology, vol. 105, no. 1, pp. 86-93, 2012.

[9] T. F. Lee and F. M. Fang, "Quantitative analysis of normal tissue effects in the clinic (QUANTEC) guideline validation using quality of life questionnaire datasets for parotid gland constraints to avoid causing xerostomia during head-and-neck radiotherapy," Radiotherapy and Oncology, vol. 106, no. 3, pp. 352-358, 2013.

[10] I. Beetz, C. Schilstra, A. van der Schaaf et al., "NTCP models for patient-rated xerostomia and sticky saliva after treatment with intensity modulated radiotherapy for head and neck cancer: the role of dosimetric and clinical factors," Radiotherapy and Oncology, vol. 105, no. 1, pp. 101-106, 2012.

[11] A. Jackson, R. Ten Haken, J. Robertson, M. Kessler, G. Kutcher, and T. Lawrence, "Analysis of clinical complication data for radiation hepatitis using a parallel architecture model," International Journal of Radiation Oncology, Biology, Physics, vol. 31, no. 4, pp. 883-891, 1995.

[12] C. J. Xu, A. Van Der Schaaf, C. Schilstra, J. A. Langendijk, and A. A. Van'T Veld, "Impact of statistical learning methods on the predictive power of multivariate normal tissue complication probability models," International Journal of Radiation Oncology Biology Physics, vol. 82, no. 4, pp. e677-e684, 2012.

[13] A. van der Schaaf, C.-J. Xu, P. van Luijk, A. A. Van't Veld, J. A. Langendijk, and C. Schilstra, "Multivariate modeling of complications with data driven variable selection: guarding against overfitting and effects of data set size," Radiotherapy and Oncology, vol. 105, no. 1, pp. 115-121, 2012.

[14] C.-J. Xu, A. van der Schaaf, A. A. van'T Veld, J. A. Langendijk, and C. Schilstra, "Statistical validation of normal tissue complication probability models," International Journal of Radiation Oncology, Biology, Physics, vol. 84, no. 1, pp. e123-e129, 2012.

[15] M. Söhn, D. Yan, J. Liang, E. Meldolesi, C. Vargas, and M. Alber, "Incidence of late rectal bleeding in high-dose conformal radiotherapy of prostate cancer using equivalent uniform dose-based and dose-volume-based normal tissue complication probability models," International Journal of Radiation Oncology Biology Physics, vol. 67, no. 4, pp. 1066-1073, 2007.

[16] A. Trotti, R. Byhardt, J. Stetz et al., "Common toxicity criteria: Version 2.0. An improved reference for grading the acute effects of cancer treatment: impact on radiotherapy," International Journal of Radiation Oncology Biology Physics, vol. 47, no. 1, pp. 13-47, 2000.

[17] I. El Naqa, J. Bradley, A. I. Blanco et al., "Multivariable modeling of radiotherapy outcomes, including dose-volume and clinical factors," International Journal of Radiation Oncology Biology Physics, vol. 64, no. 4, pp. 1275-1286, 2006.

[18] R. Tibshirani, "Regression shrinkage and selection via the lasso," Journal of the Royal Statistical Society B: Methodological, vol. 58, no. 1, pp. 267-288, 1996.

[19] T.-F. Lee, P.-J. Chao, H.-M. Ting et al., "Using multivariate regression model with Least Absolute Shrinkage and Selection Operator (LASSO) to predict the incidence of Xerostomia after intensity-modulated radiotherapy for head and neck cancer," PLoS ONE, vol. 9, no. 2, Article ID e89700, 11 pages, 2014.

[20] B. Efron, T. Hastie, I. Johnstone, and R. Tibshirani, "Least angle regression," The Annals of Statistics, vol. 32, no. 2, pp. 407-499, 2004.

[21] T. Hastie, R. Tibshirani, J. Friedman, and J. Franklin, "The elements of statistical learning: data mining, inference and prediction," The Mathematical Intelligencer, vol. 27, no. 2, pp. 83$85,2005$.

[22] A. K. Shadad, F. J. Sullivan, J. D. Martin, and L. J. Egan, “Gastrointestinal radiation injury: symptoms, risk factors and mechanisms," World Journal of Gastroentero, vol. 19, no. 2, pp. 185198, 2013.

[23] J. M. Robertson, M. Söhn, and D. Yan, "Predicting grade 3 acute diarrhea during radiation therapy for rectal cancer using a cutoff-dose logistic regression normal tissue complication probability model," International Journal of Radiation Oncology, Biology, Physics, vol. 77, no. 1, pp. 66-72, 2010. 
[24] S. C. Chang, H. F. Lee, H. M. Ting et al., "Effect of different treatment plans on irradiated small-bowel volume in gynecologic patients undergoing whole-pelvic irradiation," Journal of Radiation Research, vol. 54, no. 5, pp. 909-918, 2013.

[25] J. T. Lyman, "Complication probability as assessed from dosevolume histograms," Radiation Research, vol. 104, no. 2, pp. S13S19, 1985.

[26] A. B. Miah, S. L. Gulliford, C. H. Clark et al., "Dose-response analysis of parotid gland function: what is the best measure of xerostomia?" Radiotherapy and Oncology, vol. 106, no. 3, pp. 341-345, 2013.

[27] M. W. Skwarchuk, A. Jackson, M. J. Zelefsky et al., "Late rectal toxicity after conformal radiotherapy of prostate cancer (I): multivariate analysis and dose-response," International Journal of Radiation Oncology Biology Physics, vol. 47, no. 1, pp. 103-113, 2000.

[28] J. Xu and J. Yin, "Kernel least absolute shrinkage and selection operator regression classifier for pattern classification," Computer Vision, IET, vol. 7, no. 1, pp. 48-55, 2013.

[29] L. B. Marks, E. D. Yorke, A. Jackson et al., "Use of normal tissue complication probability models in the clinic," International Journal of Radiation Oncology, Biology, Physics, vol. 76, no. 3, pp. S10-S19, 2010.

[30] B. D. Kavanagh, C. C. Pan, L. A. Dawson et al., "Radiation dosevolume effects in the stomach and small bowe," International Journal of Radiation Oncology Biology Physics, vol. 76, no. 3, pp. S101-S107, 2010.

[31] I. Chitapanarux, A. Tonusin, V. Sukthomya, C. Charuchinda, N. Pukanhapan, and V. Lorvidhaya, "Phase II clinical study of irinotecan and cisplatin as first-line chemotherapy in metastatic or recurrent cervical cancer," Gynecologic Oncology, vol. 89, no. 3, pp. 402-407, 2003.

[32] K. N. Moore, M. W. Sill, D. S. Miller et al., "A phase I trial of tailored radiation therapy with concomitant cetuximab and cisplatin in the treatment of patients with cervical cancer: a gynecologic oncology group study," Gynecologic Oncology, vol. 127, no. 3, pp. 456-461, 2012.

[33] P. G. Rose, M. W. Sill, D. S. McMeekin et al., "A phase i study of concurrent weekly topotecan and cisplatin chemotherapy with whole pelvic radiation therapy in locally advanced cervical cancer: a gynecologic oncology group study," Gynecologic Oncology, vol. 125, no. 1, pp. 158-162, 2012. 


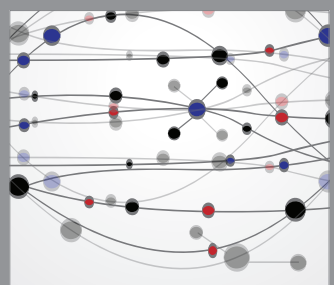

The Scientific World Journal
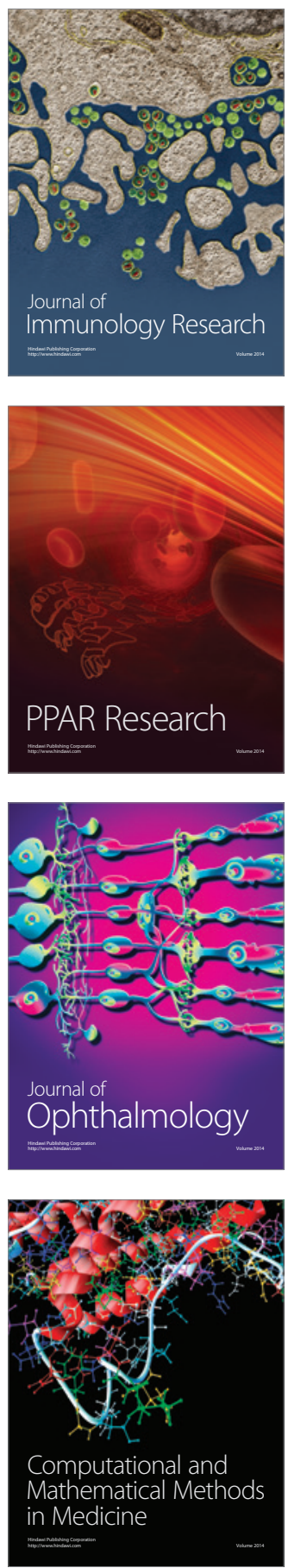

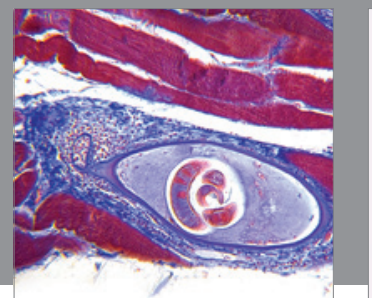

Gastroenterology

Research and Practice
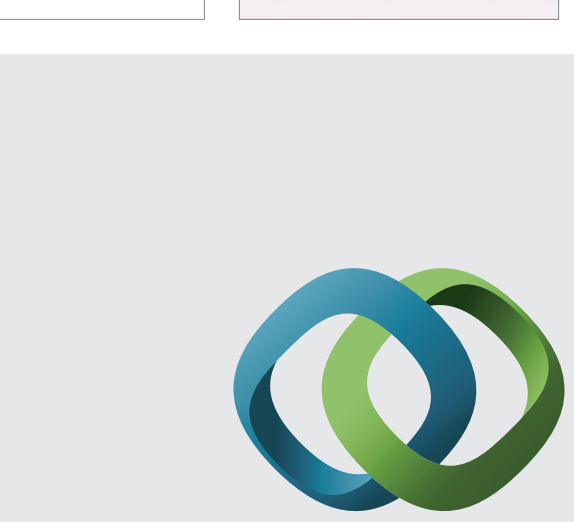

\section{Hindawi}

Submit your manuscripts at

http://www.hindawi.com
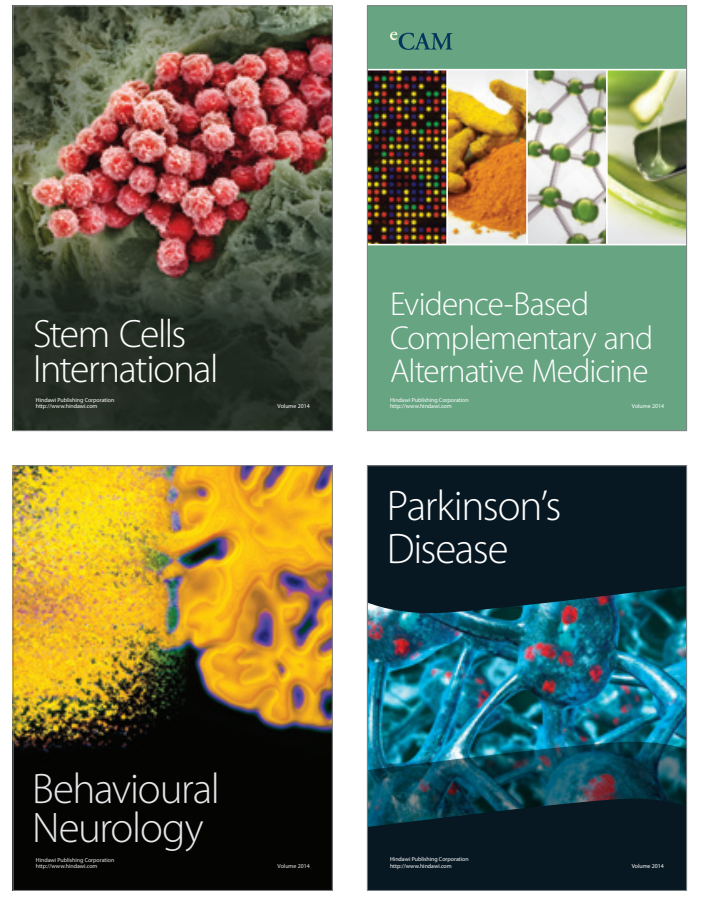
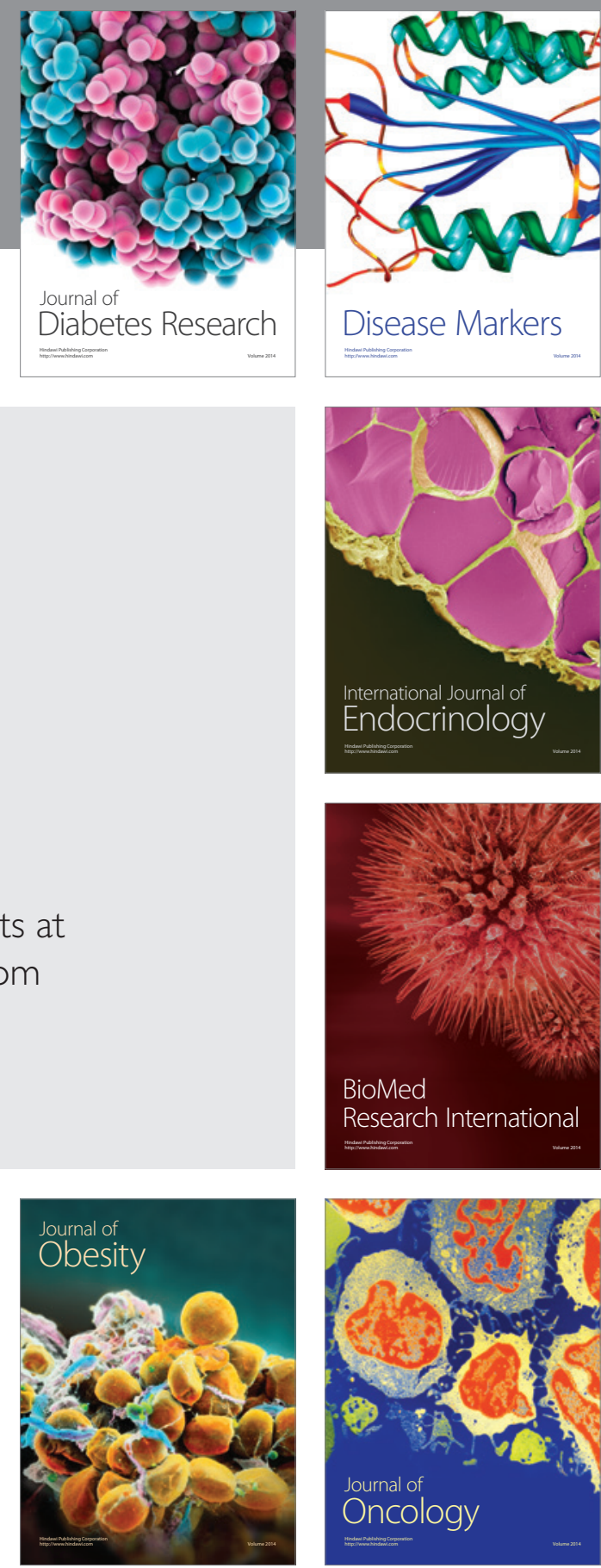

Disease Markers
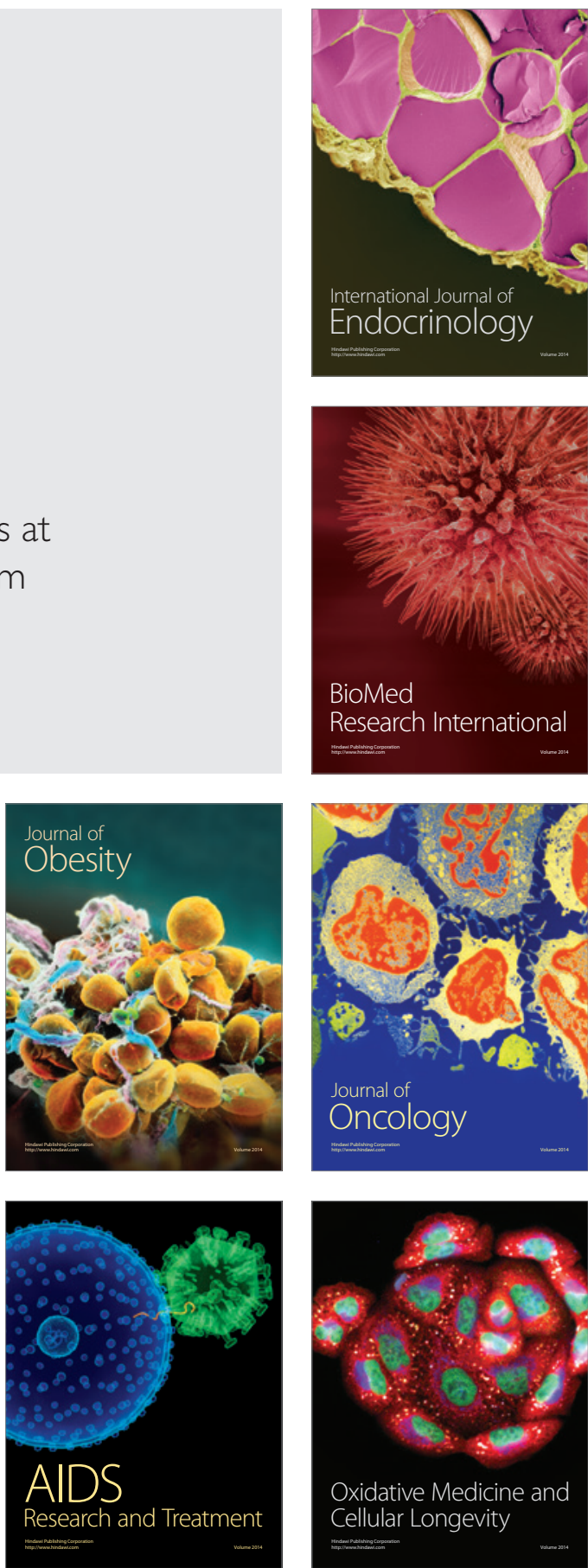